\title{
Electronics, heal thyself
}

\section{Self-healing materials could deliver a new generation of electronic devices.}

- r ectronics is a field that incorporates researchers with a diverse range of backgrounds, from physicists and nanotechnologists to electronic engineers and computer scientists. But key to the development of new electronic materials is the work of chemists. (Gordon Moore, co-founder of Intel and the eponymous definer of Moore's law, was incidentally trained as a chemist.) The role of chemistry can, for example, be seen in the advance of electronics based on two-dimensional materials and the layered two-dimensional materials known as van der Waals heterostructures. It can also be seen in the advance of self-healing electronic materials, which have recently emerged from attempts to use polymer chemistry to mimic the healing capabilities of human skin.

In a Review Article in this issue of Nature Electronics, Jiheong Kang, Jeffrey Tok and Zhenan Bao at Stanford University explore the development of self-healing electronic materials. They first consider the different ways these materials can be created, which can be split into two broad categories: extrinsic and intrinsic. Extrinsic selfhealing materials do not themselves have self-healing properties, but instead contain healing agents that are released when the material is damaged. Intrinsic self-healing materials, on the other hand, rely on the inherent reversibility of dynamic covalent or non-covalent bonds that make up their polymer matrix.

Next, the researchers explain how these materials can be used to build different electronic components, devices and integrated systems. The versatility of self-healing polymers means that it is possible to create materials that are insulators, conductors or semiconductors, and the different polymers have already been incorporated into a variety of devices, including field-effect transistors, solar cells and sensors. Demonstrations of integrated systems are still at an early stage, but a few examples have recently emerged. For instance, an integrated selfhealable electronic skin system was created in 2018 that combines a strain monitor,

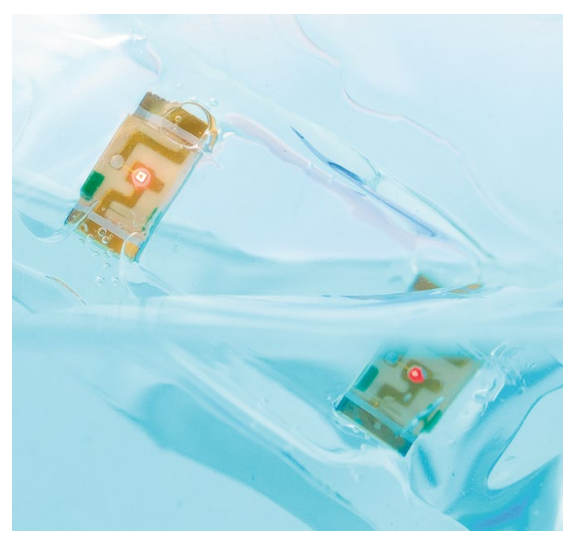

An ionic circuit board with embedded lightemitting diodes, which is made from a transparent electronic skin that can self-heal in both wet and dry conditions due to reversible ion-dipole interactions. Credit: Benjamin C.K. Tee and Wangwei Lee, National University of Singapore

an electrocardiogram sensor and a lightemitting capacitor display array ${ }^{1}$. The system, which is entirely self-healable and can be stretched by up to $100 \%$, can be used to both monitor and display a person's physiological signals.

Finally, Bao and colleagues turn to the problem of transforming self-healing electronics into a practical, everyday technology. They highlight key challenges in fabrication, properties, healing processes, healing scale and thermal stability. The challenges are considerable, but the materials can offer unique capabilities, opening up applications that would not be possible with conventional electronic systems. The intriguing possibilities of these materials were, for example, recently highlighted by the work of Chao Wang, Benjamin Tee and colleagues, who reported self-healing electronic skins that can function underwater ${ }^{2}$.

Inspired by transparent jellyfish, the researchers - who are based at institutes in China, the US and Singapore - created transparent electronic skins by combining a fluorocarbon-based polymer and a fluorine- rich ionic liquid. The resulting material is linked via reversible ion-dipole interactions, an approach that is in contrast to the dynamic bonds typically used to build selfhealing materials: namely, hydrogen bonds and metal-ligand coordination. As a result, the material can self-heal in wet conditions, including deionized water, seawater, and acidic and basic solutions, as well as in dry conditions. Tee and colleagues use the technology to build touch, pressure and strain sensors. They also show that it can be used to build printed circuit boards. Notably, and as Carmel Majidi at Carnegie Mellon University explores in an accompanying News \& Views article ${ }^{3}$, these underwater capabilities mean that the electronic skin could be of particular value in the development of underwater soft robotics.

Soft robots are built from materials like polymer gels and rubbers, rather than the hard metals and plastics that are typically used to build such machines ${ }^{4}$. This means that the robots have mechanical properties that are much more similar to biological substances, allowing them to better adapt to their surroundings and making interactions with humans easier and safer. However, using soft materials rather than hard, rigid materials means that the system is more susceptible to damage. And thus, giving them a skin-like ability to self-heal could be valuable. To date, a variety of soft robots that can function underwater have been demonstrated, including basic grippers and those inspired by animals such as fish and octopuses. Endowing these robots with electronic skin capable of self-healing would be another step in their evolution, and another challenge for chemists, physicists, nanotechnologists, electronic engineers, computer scientists and beyond.

Published online: 15 April 2019

https://doi.org/10.1038/s41928-019-0242-1

\footnotetext{
References

1. Son, D. et al. Nat. Nanotechnol. 13, 1057-1065 (2018).

2. Cao, Y. et al. Nat. Electron. 2, 75-82 (2019).

. Majidi, C. Nat. Electron. 2, 58-59 (2019).

4. Rich, S. I., Wood, R. J. \& Majidi, C. Nat. Electron. 1, 102-112 (2018).
} 